\title{
Thermal Characteristic Analysis for The Spindle System of EDM
}

\author{
Li Fuping ${ }^{1 ; a, \text { Meng Zhaoyang }}{ }^{2 ; b}$,Liu Zhifeng ${ }^{3 ; c}$, Liu Jianyong ${ }^{4 ; d}$ \\ 1,2,3Beijing Key Laboratory of Advanced Manufacturing Technology,Beijing University of \\ Technology,Beijing 100124 China \\ ${ }^{4}$ Beijing Institute of Electro Machining,Beijing 100191 China \\ alifp@bjut.edu.cn, ${ }^{b} 286183669 @ q q . c o m,{ }^{c}$ lzf@bjut.edu.cn, ${ }^{\text {d20904736@qq.com }}$
}

Keywords:EDM,Spindle thermal deformation, thermal-structure coupling deformation, temperature field.

\begin{abstract}
The spindle system of EDM is directly involved in the processing process, and its thermal deformation seriously affect the processing accuracy.In this paper,we can use the method of sequential coupling,which based on the software of Workbench ANSYS,to analyze the characteristics of thermal-structure coupling in the spindle system. The temperature field and thermal deformation of the spindle system have been calculated by the simulation. And the results of finite element analysis have been verified by experiments. The results show that the internal heat source of the spindle system has a serious influence on the temperature rise and thermal deformation, and the maximum thermal deformation occurred in the direction of $\mathrm{X}$ axis, while the thermal deformation of other direction is not significant to the thermal deformation of the spindle system. The analysis results lay the foundation for further improving the temperature field of the spindle system and reducing the thermal deformation, and it also provides a strong basis for the design of optimization and error compensation of the spindle system.
\end{abstract}

\section{Introduction}

Electric Discharge Machine(EDM) has widely used in aviation,aerospace,automobile manufacturing and mold manufacturing industry and other related industries.In today's manufacturing field,It's development of products with high precision, material diversification and processing complexity, and it has put forward a higher requirement for EDM machine ${ }^{[1]}$. Because of the combination of internal and external heat source in the working process, and result in uneven temperature distribution in the machine,the machine parts occurred different extent of thermal deformation, which destroy the relative motion between the machine tool parts, reduce the machining accuracy of machine tools.According to statistical studies, in precision machining, the manufacturing error caused by the thermal deformation of the machine is $40 \% \sim 70 \%{ }^{[2]}$. Therefore, it is important to analyze the thermal characteristics of EDM, and then to reduce the thermal deformation of it, which is of great significance to improve the machining accuracy.Thermal deformation of machine tool spindle system is the main factor affecting machining accuracy of EDM .Due to the complex structure of the spindle system of the machine tool, it is difficult to establish a mathematical model to calculate its deformation, and the finite element analysis is an effective means to study the deformation of the complex structure.

The main heat source of this paper includes the friction heat of the bearing, the friction between the lead screw and the nut, the friction between the guide rail and the sliding block, and the heat of the motor.Considering the screw rotation to drive the surrounding gas, which can result in the phenomenon of forced convection, and based on a large EDM, then initial and boundary conditions are established for the analysis of thermal characteristics of EDM's spindle system,as shown in Fig.1.The finite element model of the spindle system is established by using SolidWorks and 
Workbench ANSYS software, and the Thermal-structure coupling analysis is carried out on the basis of this model. We can through the analysis of the thermal deformation of the spindle to explore the influence factors of thermal deformation of the spindle system, and we can verify the accuracy of the model analysis to provide a strong basis for the improvement of the structure in the future.

\section{The establishment of finite element model}

This article based on the SolidWorks software to build the three-dimensional simplified model into the Workbench ANSYS software.First,Setting the material properties of each component (such as density, elastic modulus, Poisson's ratio, thermal conductivity, thermal expansion coefficient, etc.).The spindle system model is hexahedral mesh generation by using the method of combination of automatic and manual mesh,and then we obtained 158800 nodes and 80612 units. The generated finite element model is shown in Fig.2.

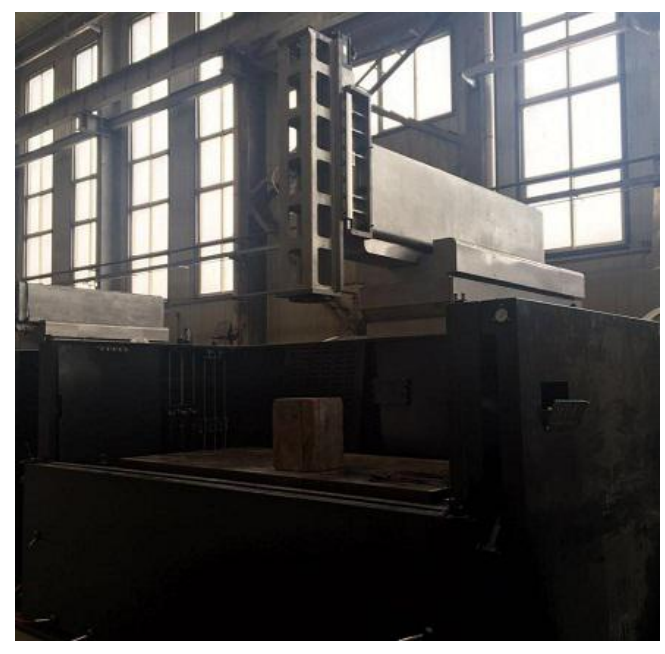

Fig.1 a large EDM

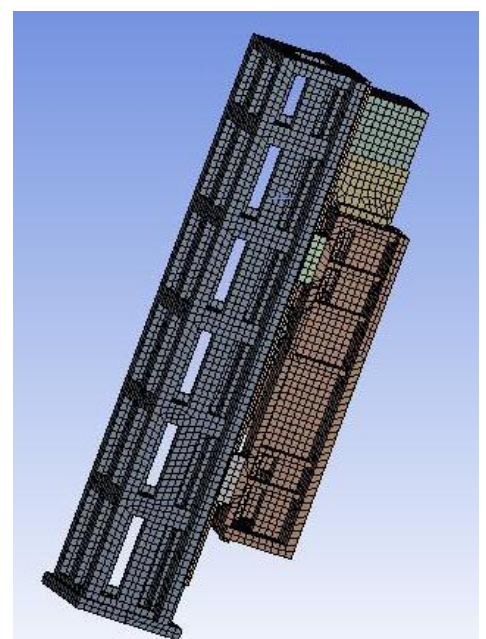

Fig.2 Finite element model of spindle system

\section{Calculation of heat source and boundary conditions of spindle system}

When the spindle speed is $600 \mathrm{r} / \mathrm{min}$,we calculate the main heat source which affects the thermal deformation of the spindle,including the friction heat of the bearing,the friction between the lead screw and the nut,the friction between the guide rail and the sliding block,and the heat of the motor.Because of the rotation of the lead screw, it will drive the surrounding gas movement and then result in forced convection and natural convection heat.We can explore the main factors affecting the accuracy of the spindle.

Servo motor heating. Motor model is Panasonic of 3KW MGME.Assuming that the working process of the machine tool, the motor rated power loss all converted into heat energy,so it's heat generation $\left(Q_{m}\right)$ is as follows:

$$
Q_{m}=P \times(1-\eta)=\frac{M_{T} \cdot \mathrm{n}}{9550} \times(1-\eta)
$$

In the formula(1):P is motor input power; ${ }^{M_{T}}$ is output torque; $\eta$ is motor efficiency.

Friction heat of bearing.Bearing friction heat is related to friction torque and rotational speed,According to the reference ${ }^{[5]}$, the calculation formula of bearing heat source is as follows: 


$$
\left\{\begin{array}{l}
Q_{\mathrm{b}}=1.047 \times 10^{-4} M \mathrm{n} \\
M=M_{1}+M_{0}
\end{array}\right.
$$

In the formula(2): $Q_{b}$ is bearing friction heat; $n$ is speed; $M$ is total friction torque; ${ }^{M_{1}}$ is friction torque caused by load; ${ }^{M_{0}}$ is viscous friction torque.

Friction torque $\left(M_{1}\right)$ is related to the bearing load,reflecting the friction loss of elastic hysteresis and local sliding;Viscous friction torque $\left(M_{0}\right)$ is related to speed,reflecting the fluid power loss of lubricant.The calculation formulas of these are as follows:

$$
M_{1}=f_{1} P d_{m}
$$

In the formula(3): $f_{1}$ is coefficient related to bearing type and load; $P$ is calculation load of bearing friction torque, and it mainly depends on the magnitude and direction of the force; ${ }_{m}$ is middle diameter of bearing.

In this paper,Selection of bearings are angular contact ball bearings, and the calculating formula of $f_{1}$ and $P$ are shown in Table 1 .

Table 1 Correlation parameter formula

\begin{tabular}{ccc}
\hline & $f_{1}$ & $P$ \\
\hline Single row & $0.0013\left(F_{0} / C_{0}\right)^{0.33}$ & $F_{a}-0.1 F_{r}$ \\
Double row & $0.001\left(F_{0} / C_{0}\right)^{0.33}$ & $1.44 F_{a}-0.1 F_{r}$ \\
\hline
\end{tabular}

In these formula: ${ }^{F_{0}}$ is equivalent static load; ${ }^{F_{a}}$ is axial load; ${ }^{C_{0}}$ is basic static load rating; $F_{r}$ is radial load.

When $m \geq 2000$, the calculating formula of $M_{0}$ is as follows:

$$
M_{0}=10^{-7} f_{0}(v \mathrm{n})^{\frac{2}{3}} \mathrm{~d}_{\mathrm{m}}^{3}
$$

When $m<2000$, the calculating formula of $M_{0}$ is as follows:

$$
M_{0}=160 \times 10^{-7} f_{0} \mathrm{~d}_{\mathrm{m}}^{3}
$$

In the formula(4,5): $f_{0}$ is coefficient related to bearing type and Lubrication mode. When the angular contact ball bearings are single row and grease lubrication, $f_{0}=2$. And when the angular contact ball bearings are double row and grease lubrication, $f_{0}=4 . v$ is kinematic viscosity of 
lubricant at operating temperature.

Friction heat of ball screw.Heating of the nut of the ball screw is proportional to friction torque $(T)$ and speed $(n)$.According to the reference ${ }^{[6]}$, the calculation is as follows:

$$
Q_{b}=0.12 \pi n T
$$

In the formula(6): $Q_{b}$ is heat capacity of ball screw per unit time(W); ${ }^{n}$ is rotational speed of ball screw $(\mathrm{r} / \mathrm{min}) ; T$ is friction torque of $\operatorname{nut}(N \cdot m)$.

The calculation method of friction torque of ball $\operatorname{screw}(T)$ is as follows:

$$
T=2 z\left(T_{g}+T_{e}\right) \cos \beta
$$

In the formula(7):z is number of rolling elements; $\beta$ is helix angle of lead screw( $\mathrm{rad}){ }^{T_{g}}$ is geometric sliding friction torque $(N \cdot m) ;{ }_{e}$ is friction resistance moment $(N \cdot m)$.

The related calculation formulas are as follows:

$$
\begin{aligned}
& T_{g}=0.08 \frac{f m_{\alpha}^{2}}{R} \sqrt[3]{\frac{16 Q^{5}}{\left(\vartheta \sum \rho\right)^{2}}} \\
& T_{e}=m_{\rho} \sqrt[3]{\frac{4 Q^{4}}{\vartheta \sum \rho}} \\
& \vartheta=\frac{8}{3\left(\frac{1-\mu_{1}^{2}}{E_{1}}+\frac{1-\mu_{2}^{2}}{E_{2}}\right)}
\end{aligned}
$$

In the formula $(8,9,10):{ }^{f}$ is sliding friction coefficient; $m_{\alpha}$ and $\quad m_{\beta}$ are coefficient related to eccentric ratio of deformation ellipse of spherical contact; $Q$ is radial pressure on a single sphere $(\mathrm{N}) ; E_{1}$ and $E_{2}$ is elastic modulus of material $\left(N / \mathrm{mm}^{2}\right) ; \mu_{1}$ and $\mu_{2}$ is poisson's ratio of material. $R=R_{1} R_{2} /\left(R_{1}+R_{2}\right) \quad, \quad R_{1} \quad$ and $\quad R_{2} \quad$ are curvature radius of ball and roller; $\sum \rho=\rho_{11}+\rho_{12}+\rho_{21}+\rho_{22}, \rho_{11} 、 \rho_{12} 、 \rho_{21} 、 \rho_{22}$ are the main curvature of two rolling bodies.

Heat of the guide rail.Each guide rail is provided with two heat sources which are the frictional heat of rail and two slides.Due to the movement of the sliding block, the two heat sources are continuously moving, and the moving heat source is called the moving heat source.According to the reference ${ }^{[7]}$,the heat caused by friction is calculated as follows:

$$
Q=\frac{\mu F v}{J}
$$

In the formula(11): $\mu$ is dynamic friction coefficient; $F$ is load applied on the friction 
surface $(\mathrm{N}) ; J$ is mechanical equivalent of heat,its value is $4.2 \mathrm{~J} / \mathrm{cal} ; v$ is sliding speed of sliding block relative to the slide $\operatorname{rail}(\mathrm{m} / \mathrm{s}) ; Q$ is calorific value $(\mathrm{J} / \mathrm{s})$. The friction coefficient is 0.05 , and finally the heat flux of the contact surface between a single guide rail and a sliding block is $492.5 \mathrm{w} / \mathrm{m}^{2}$.

Calculation of heat transfer coefficient. The outer surface of the machine tool is in contact with the fluid, and it is the heat exchange, which is the main heat release form of the machine tool.According to the Nusselt criterion, calculation formula of heat transfer coefficient is as follows:

$$
h=\frac{N u \cdot \lambda}{L}
$$

In the formula(12): $N u$ is Nusselt number; $L$ is feature size; $\lambda$ is heat transfer coefficient of fluid.

As we can see from the above equation that if the Nusselt number is solved,we can calculate the corresponding coefficient of convective heat transfer.According to the reference ${ }^{[6,8]}$, the standard equation of natural convection heat transfer is as follows:

$$
\begin{aligned}
& N u=C(G r \cdot \operatorname{Pr})_{m}^{n} \\
& G r=\frac{g \beta L^{3} \Delta t}{V^{2}} \\
& \operatorname{Pr}=\frac{V}{a}
\end{aligned}
$$

In the formula $(13,14,15): C, n$ is constant, ${ }_{r}$ is Grashof numeral; ${ }^{P}$ is Prandtl number; ${ }^{g}$ is gravitational acceleration; $\beta$ is coefficient of expansion; $v$ is kinematic viscosity.By calculating the Nusselt number, then we can obtain the natural convection heat transfer is $27 \mathrm{~W} /\left(\mathrm{m}^{2} \cdot{ }^{\circ} \mathrm{C}\right)$.

When lead screw rotates at a certain speed, it will speed up the convection of the air.Because the lead screw is provided with a spiral groove,the convection heat transfer efficiency of the lead screw is larger than that of the diameter optical rod.The criterion equation of the convection heat transfer of the rotating shaft is as follows:

$$
N u=0.133 \operatorname{Re}^{2 / 3} \operatorname{Pr}^{1 / 3}
$$

In the formula(16): $\operatorname{Re}$ is Reynolds Number, $\operatorname{Re}=\frac{\omega \cdot d}{v} ; \omega$ is the angular velocity of ball screw; $d$ is diameter of ball screw.In the same way, the forced convective heat transfer is $73.9 \mathrm{~W} /\left(\mathrm{m}^{2} \cdot{ }^{\circ} \mathrm{C}\right)$.

\section{Test verification and result analysis}

The heat flux and boundary conditions obtained from the above calculation are applied to the finite element model which has been partitioned.By steady-state thermal analysis, the temperature field map is obtained as shown in Fig.3(Temperature field of spindle system). 


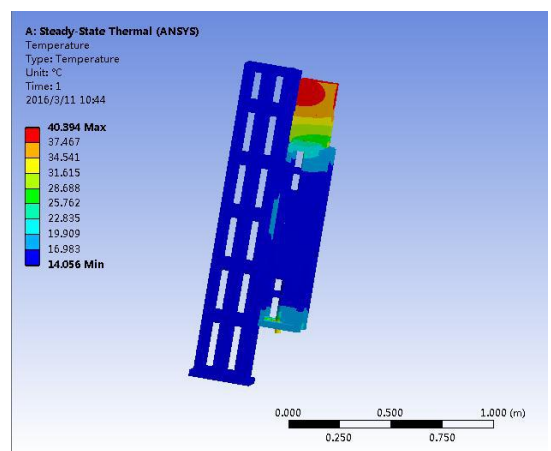

Fig.3(a)

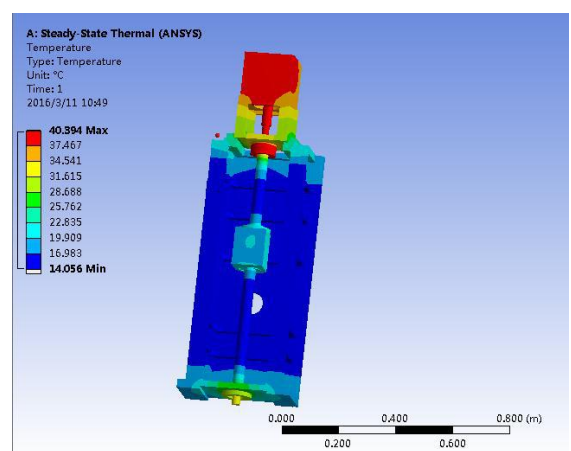

Fig.3(b)

Through the map of temperature field,we can clearly see the parts of the main spindle which generated heat.In order to verify the accuracy of the simulation results, the temperature measurement of each component of the machine tool is carried out.Specific operations are as follows:we let the spindle of the machine tool run at speed of $600 \mathrm{r} / \mathrm{min}$ at the temperature of $14^{\circ} \mathrm{C}$, and when spindle system has achieved thermal balance.Then we use thermal imaging instrument to measure the main spindle system. We average temperature of each component, and the results are shown in Fig.4.
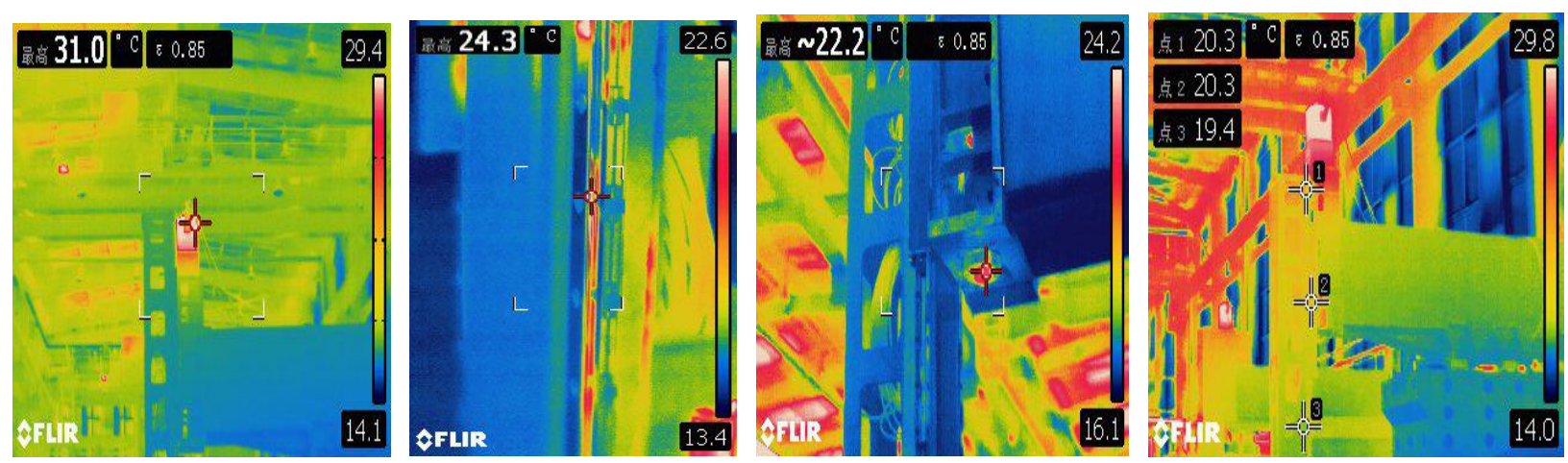

Fig.4 Temperature of each component of the spindle system

Comparison between measurement data of thermal imaging instrument and the corresponding parts of simulation of temperature field,we can get the results as shown in Fig.5.
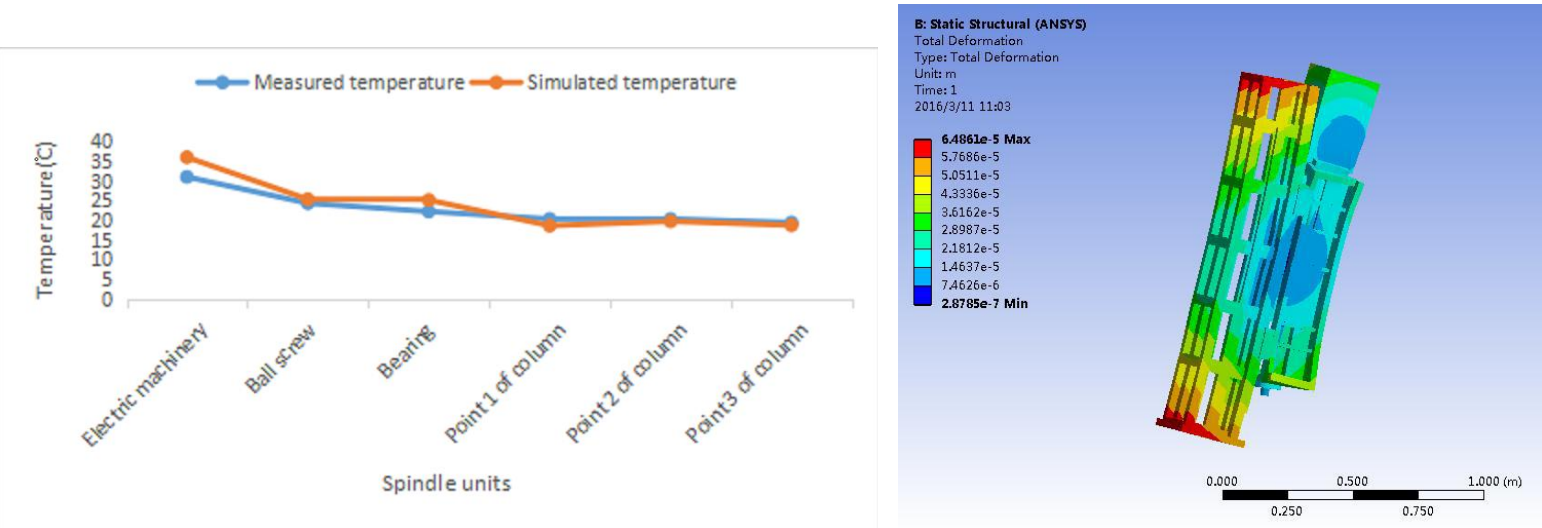

Fig.5 Comparison result

Fig.6 Thermal deformation of the spindle system

As we can see in Figure 5,because the measured datum are consistent with the simulated results, the accuracy of the simulation can be proved.And we can analyze the temperature field as the load on the final thermal deformation of the spindle system of the machine tool.The static analysis is carried out by adding load of temperature field,load of gravity and fixed constraint,and the total thermal deformation is shown in Fig.6. 


\section{Conclusion}

We have carried out the finite element analysis of the spindle system of EDM,and obtained the distribution of temperature field and thermal deformation of the spindle system. The correctness of the finite element model is verified by experiments. The final conclusion is as follows:

(1)The finite element analysis which can analyze the thermal characteristics of the spindle system is a reliable and effective method.

(2)As we can see through the temperature of the spindle system, its main heat source is the heating of the motor, bearings and ball screw.However, due to the existence of the motor seat, which can effectively isolate the heat from the motor,the heat source is mainly concentrated in the bearing and the ball screw.The temperature field distribution of the system,which is uneven, is easy to cause the deformation of the spindle system. So we can improve the temperature distribution characteristics of the spindle system by reducing the intensity of the heat source and strengthening the heat dissipation.

(3)As we can see through the thermal deformation of spindle system,in the bottom of the column this part,where is the position of the electrode position, has achieved the maximum deformation of $64.81 \mu m$, and the deformation of $\mathrm{X}$ axis is $64.1 \mu m$, the deformation of $\mathrm{Y}$ axis is $17.68 \mu m$, the deformation of $\mathrm{Z}$ axis is $21.97 \mu \mathrm{m}$. So the thermal deformation of the spindle system is mainly concentrated in the direction of $\mathrm{X}$ axis.

the thermal deformation will have a certain impact on the machining accuracy of EDM, and then it will affect the quality of machining. So it is very important to take certain measures to prevent and control the thermal deformation of $\mathrm{X}$ axial to reduce the influence of thermal deformation on the machining accuracy.The analysis of the temperature field and the thermal deformation distribution of the spindle system has laid the foundation for the further improvement of the characteristics of temperature distribution and the reduction of the thermal deformation.

\section{Acknowledgements}

Major national science and technology projects,Jing Hua talent project of BJUT (2014ZX04010-011);

Major national science and technology projects,Scale promotion project of high-grade domestic CNC grinding machine reliability(2013ZX04011-013).

\section{Reference}

[1]Yang Dayong,Fu Jinjuan.Technology and development trend of EDM[J].Aviation manufacturing technology.2010(5):43-46.

[2]Ramesh R.E1T or Compensation in machine tools-a review part 1:thermal errors[J].International Journal of Machine Tools and Manufacture.2000(40):1257-1284.

[3]Liu Xianghua,Wen Lihua,Li Xiaona.thermal-structural coupling analysis of missile wing of short-range hypersonic[J].Computer aided engineering.2009(12):67-69.

[4]Li Weite,et al.Theoretical analysis and application of thermal stress[M].China Electric Power Press(Beijing).2004.

[5]Wang Jinsheng.Study of thermal characteristics on the XK717 CNC milling machine[D].Master Thesis of Zhejiang University of Technology.2004(5):15-31.

[6]He Zhen.Thermal analysis and thermal deformation compensation for ball screw system of machine tools[D].Southwest Jiaotong University Master Degree Thesis.2009:26-28. 
[7]Sun Zhili,YANG Qiang. Study on thermal characteristics of slide guide of the machine tool based on FEM[J].Journal of Northeastern University,2011,32(7):1000-1003.

[8]Yang Shiming,Tao Wenquan.Heat transfer(Fourth Edition)[M].Higher Education Press(Beijing), 2006. 